\title{
A novel scoring system for vestibular schwannomas to identify candidacy for cochlear implantation
}

\author{
Christoph Arnoldner ${ }^{1}$, Ursula Schwarz-Nemec ${ }^{2}$, Alice Auinger ${ }^{3}$, Erdem Yildiz $^{3}$, Christian \\ Matula $^{3}$, and Valerie Dahm ${ }^{3}$ \\ ${ }^{1}$ Medizinische Universitat Wien \\ ${ }^{2}$ Medizinische Universitat Wien Universitatsklinik fur Radiologie und Nuklearmedizin \\ ${ }^{3}$ Medical University of Vienna
}

February 19, 2021

\section{Key points}

- Simultaneous translabyrinthine tumor resection and cochlear implantation is a promising treatment method with hearing rehabilitation for sporadic vestibular schwannomas.

- Size of vestibular schwannoma, distance to the modiolus, residual hearing and promontory stimulation electrical brainstem response audiometry are important preoperative predictive factors for chance of cochlear implantation.

- Electrically evoked brain stem response audiometry with an intracochlear test electrode is an important additional tool during vestibular schwannoma resection to decide on cochlear implantation.

Sporadic vestibular schwannomas (VS) can be treated using radiation therapy, microsurgical resection, or a wait-and-scan approach. Most VS will require treatment after some period of observation. However, the currently available treatment options carry a high risk of loss of serviceable hearing. Some authors believe that the possibility of cochlear implantation in the setting of VS should completely change the treatment strategy for this disease(1). However, patient outcomes with CIs show a wide variation - with some patients having no auditory perception, while others exhibit open-set speech understanding(2). The intraoperative testing of cochlear nerve function using electrically evoked brainstem response audiometry (eABR) provides a means of objectively assessing cochlear nerve conduction, and has been increasingly applied in this setting $(2$, 3 ). In the present study, we aimed to assess outcomes of patients undergoing vestibular schwannoma resection and cochlear implantation, and to develop a new scoring system to preoperatively identify suitable patients for this treatment course. Appropriate preoperative classification of patients can have important impacts on patient counseling and expectations.

\section{Design}

Each study participant gave their informed consent to participate. The trial was approved by the local ethics committee 1111/2017, and is registered at www.clinicaltrials.gov (NCT03745560). 
All 17 patients underwent translabyrinthine vestibular schwannoma resection, during which eABR were recorded with an intracochlear test electrode (ITE) before and after tumor resection. In cases with positive responses after complete tumor resection, a cochlear implant (CI) was placed. Overall, ten patients received a CI with a Flex 28 or Flex Standard electrode (MED-EL, Innsbruck, Austria) depending on cochlear duct length.

\section{Data availability statement}

The data that support the findings of this study are available from the corresponding author upon request.

\section{Setting}

The study was conducted at a tertiary care center.

\section{Participants}

Seventeen patients with unilateral sporadic VS were screened between January 2017 and January 2020 (see Table 1). Inclusion criteria were as follows: sporadic vestibular schwannoma, no ipsilateral functional hearing, desire to undergo cochlear implantation, and general good health. Exclusion criteria were prior treatment for vestibular schwannoma, neurofibromatosis type 2 , history of pathology requiring multiple head magnetic resonance imaging (MRI), or history of malignant disease of the head and neck. A lack of functional hearing was defined as [?]50\% monosyllable recognition at $80 \mathrm{~dB}$ HL.

\section{Main outcome measures}

The patients' preoperative findings and results were re-evaluated to develop a new scoring system for patient selection. All patients underwent MRI of the brain performed using a 3.0 Tesla magnetic resonance (MR) unit (Philips Achieva; Philips Medical Systems, Best, the Netherlands). To visualize the cerebellopontine angle, the MR protocol included a 3D balanced fast field echo sequence that was used for further assessment. Using a picture archiving and communication system (IMPAX; AGFA HealthCare, Bonn, Germany), all MR examinations were anonymized and randomly presented to a neuroradiologist who was not aware of any clinical data. Grading according to four-grade Koos classification system was carried out (4). Additionally, a four level grading system was used to evaluate intrameatal extension towards the modiolus (Table 2).

Promontory stimulation eABR was performed with a gold coated rigid electrode with a rounded-bent (hockey stick) tip stimulation probe manufactured by MED-EL (Innsbruck, Austria) and recorded with the Neuropack, Nihon Kohden, Tokyo. A positive response is defined as a clear wave V reproducible in latency and amplitude. In cases with a wave $\mathrm{V}$ that can only be seen at high stimulation intensities or that is not reproducible an unclear response is documented. No identifiable wave $\mathrm{V}$ is defined as a negative response.

The pure-tone average (4-PTA) was calculated as the mean for each patient, using the results at frequencies of $0.5,1,2$, and $4 \mathrm{kHz}$. Monosyllables were measured at 65 and $80 \mathrm{~dB}$ HL. Based on these results, patients were categorized in four groups as seen in Table 2. STARD guidelines were followed when reporting the data of this study.

\section{Results}

After six months of follow-up, ten patients were daily users of the CI. Their mean aided pure-tone average was $38 \mathrm{~dB}$ HL, and their mean WRS was $28 \%$ at $65 \mathrm{~dB}$, and $52 \%$ at $80 \mathrm{~dB}$. Outcomes for individual patients can be seen in Table 3. Nine of the ten patients had open-set speech understanding. The patient without open-set speech understanding has significant hearing loss on the contralateral side and a language barrier. 
Facial nerve outcomes according to House Brackman (HB) are shown in Table 3. In all cases, the six-month follow-up MRI showed no sign of residual or recurrent tumor.

\section{The scoring system}

Table 2 shows the scoring system that was developed. Besides Koos grading, results of promontory stimulation eABR were included. Our system also evaluated residual hearing, which is an important factor reflecting the state of the nerve and the cochlea before an intervention. Further, we identified relation to the modiolus as a predictive factor. Table 4 shows the scoring system applied to the presented patients.

\section{Discussion}

Various advancements over recent years have made CI a good option for restoring binaural hearing in patients with VS. Imaging sequences have been improved and CI magnets have been developed to reduce metal artifacts(5) and allow postoperative evaluation of the internal auditory canal and cerebellopontine angle for tumor follow-up(6). Another recent development is the possible use of intraoperative eABR with an ITE, enabling objective assessment of cochlear nerve functionality $(2,3)$.

In the past, the outcome of CIs in the context of VS was considered unpredictable, and many patients did not benefit from this technique. However, the advent of testing systems, such as intraoperative eABR, permits more precise evaluation of possible outcomes. Although simultaneous VS resection and cochlear implantation is possible with only visual assessment of the cochlear nerve(7), eABR measurements are a useful tool for objectifying the surgeon's assessment. Only two prior studies have described the use of eABR during translabyrinthine VS resection $(2,8)$. One study included eight patients(8). The other was a pilot study by our research group(2). In this previous manuscript, we demonstrate the intraoperative objective evaluation of the cochlear nerve with eABR, using an ITE, during translabyrinthine VS resection and cochlear implantation(2). Our preliminary results indicated that positive eABR results (clear wave V) seem to be reliable, and to correlate well with CI-aided auditory perception. This thesis was further supported by the results of our present study, in which all patients with positive eABR results and who received an implant showed auditory perception with their CI, and most (90\%) even have open-set speech understanding.

A remaining major challenge is to identify patients with a high chance of cochlear nerve preservation. Therefore, as a logical next step, the knowledge gained in this study was used to create a grading system with the aim of preoperatively determining candidacy, as was performed herein.

Individuals with residual speech understanding were more likely to have positive eABR results, and to receive a CI. These results are in line with the findings of Sanna et al(7). In their study, individuals with good functional hearing underwent translabyrinthine VS resection and cochlear implantation. After tumor removal, surgeons evaluated the cochlear nerve and visually determined whether it was intact(7). Of the nineteen included patients, thirteen were able to receive a CI, of whom $84 \%$ use their CI daily or almost daily $(7)$.

Another identified predictive factor was the VS extension towards the cochlear modiolus. This anatomic structure also reportedly plays an important role when assessing CI candidacy in cases of malformation,(9) as well as the need of its preservation in intracochlear $\operatorname{VSs}(10)$. Obviously, tumor resection is increasingly challenging with greater VS size, reducing the possibility of nerve protection reflected by Koos grading. The last category of our scoring system was promontory stimulation. One issue with promontory stimulation is that it produces a substantial number of artefacts in awake patients. Another problem is that some patients do not tolerate stimulation well, leading to a wide variety of stimulations intensities between different individuals. Artefacts and said stimulation range diminish the interpretation possibilities and reliability of the results.

One limitation of the present study was the relatively small sample size on which the scoring system was based. Nevertheless, this study represents the largest group of patients with vestibular schwannomas, in 
whom eABR was intraoperatively performed. As well as the first study assessing predictive factors for cochlear implantation which can be assessed preoperatively.

Overall, our results indicated that patients with a Class I scoring had a very high chance of receiving a CI. Among patients categorized as Class II (6- 8 points according to our system), the majority could be implanted, but they had a distinctly worse chance of receiving an implant, and thus careful counseling is essential in these cases. Patients categorized as Class III and IV were not able to be implanted with a CI.

\section{Conclusion}

Simultaneous translabyrinthine VS excision and cochlear implantation using intraoperative eABR measurements is a good option for hearing rehabilitation and provides binaural hearing. Preoperative accurate assessment of the size and extent of VS, audiometric testing, and promontory stimulation eABR improves preoperative patient selection, help manage patient expectations, and predict the possibility of cochlear implantation.

\section{References}

1. Upadhyay U, Almefty RO, Dunn IF, Al-Mefty O. Letter to the Editor: Save the nerve. J Neurosurg. 2015;123(3):821-2.

2. Dahm V, Auinger AB, Honeder C, Riss D, Landegger LD, Moser G, et al. Simultaneous Vestibular Schwannoma Resection and Cochlear Implantation Using Electrically Evoked Auditory Brainstem Response Audiometry for Decision-making. Otol Neurotol. 2020;41(9):1266-73.

3. Patel NS, Saoji AA, Olund AP, Carlson ML. Monitoring Cochlear Nerve Integrity During Vestibular Schwannoma Microsurgery in Real-Time Using Cochlear Implant Evoked Auditory Brainstem Response and Streaming Neural Response Imaging. Otol Neurotol. 2020;41(2):e201-e7.

4. Koos WT, Day JD, Matula C, Levy DI. Neurotopographic considerations in the microsurgical treatment of small acoustic neurinomas. J Neurosurg. 1998;88(3):506-12.

5. Schwartz N, Rooth MA, Dillon MT, O'Connell BP, Dedmon MM, Huang BY, et al. MRI surveillance following concurrent cochlear implantation in cases of vestibular schwannoma resection. Am J Otolaryngol. 2020;41(4):102518.

6. Schwarz-Nemec U, Dahm V, Arnoldner C. Letter to the editor regarding worldwide 1st MED-EL Mi1200 SYNCHRONY cochlear implant magnet removal for MRI image artifact reduction by Wieser et al. Otolaryngology Case Reports. 2019;10(March):43-4.

7. Sanna M, Medina MD, Macak A, Rossi G, Sozzi V, Prasad SC. Vestibular Schwannoma Resection with Ipsilateral Simultaneous Cochlear Implantation in Patients with Normal Contralateral Hearing. Audiol Neurootol. 2016;21(5):286-95.

8. Roberts S, Levin B, Sanli H, Ferch R, Kong K, Eisenberg R. Simultaneous cochlear implantation and removal of acoustic neuroma: implications for hearing. J Laryngol Otol. 2020;134(6):519-25.

9. Grover M, Sharma S, Preetam C, Gupta G, Samdani S, Agarwal S, et al. New SMS classification of cochleovestibular malformation and its impact on decision-making. J Laryngol Otol. 2019;133(5):368-75.

10. Plontke SK. An Improved Technique of Subtotal Cochleoectomy for Removal of Intracochlear Schwannoma and Single-stage Cochlear Implantation. Otol Neurotol. 2020;41(7):e891. 


\begin{tabular}{l}
\hline Patient Demographics \\
ID \\
1 \\
2 \\
3 \\
4 \\
5 \\
6 \\
7 \\
8 \\
9 \\
10 \\
11 \\
12 \\
13 \\
14 \\
15 \\
16 \\
17 \\
Total \\
\hline
\end{tabular}

Table 1: Age is given in years (y). Gender is described as $\mathrm{f}$ (female) or $\mathrm{m}$ (male). Puretone average (4-PTA) is calculated as average decibel (dB) hearing level (HL) at the frequencies $500 \mathrm{Hertz}(\mathrm{Hz}), 1000 \mathrm{~Hz}, 2000 \mathrm{~Hz}$ and $4000 \mathrm{~Hz}$. Word recognition scores (WRS) are calculated using Freiburg monosyllables at (@) 80 dB HL. Hearing of the contralateral side resulted in the diagnoses of single sided deaf (SSD), asymmetric hearing loss (AHL) or bilateral complete hearing loss larger than $100 \mathrm{~dB}$ HL ( $100 \mathrm{~dB})$. Contralateral 4-PTA = 4-PTAC

Scoring System
Category
Koos Grading
Koos 3
Koos 2
Koos 1
Extension
Infiltration of modiolus
Contact with modiolus


No contact, no infiltration of modiolus

Hearing

$80<100 \mathrm{~dB} 4$-PTA

$<79 \mathrm{~dB} 4$-PTA

$>0 \%$ monosyllables, any PTA

PS EABR

Unclear wave $\mathrm{V}$

Stable wave V

Total Score

Class III

Class II

Class I

Table 2: Scoring system to identify patients with higher chances of nerve integrity in case of VS resection. A certain amount (0-3) of points are given in four categories. Points are added up and patients are categorized to a certain class which reflects the probability of cochlear implantation after translabyrinthine vestibular schwannoma excision. Promontory stimulation eABR (PS EABR)

\section{Patient Outcomes}

ID

$1 / 2 / 3 / 4$

1

2

3

4

5

6

7

8

9

10

11

12

13

14

15

16

17 
Table 3: Outcomes of seventeen included patients. The second column shows the size and extension of the vestibular schwannoma according to Koos grading one to four. The third column shows which patients were provided with a cochlear implant (CI) (yes) and which not (no). Column four - Puretone average (4-PTA) in CI aided condition calculated as average decibel (dB) hearing level (HL) at the frequencies 500 Hertz (Hz), $1000 \mathrm{~Hz}, 2000 \mathrm{~Hz}$ and $4000 \mathrm{~Hz}$. Column five and six - word recognition scores (WRS) in CI aided condition are calculated using Freiburg monosyllables at (@) 65 and $80 \mathrm{~dB}$ HL. The last two columns show facial nerve function according to House Brackmann (HB) scale 1 to 6 on postoperative day one (POD 1) and at the 6 months follow-up appointment (6 Mo FU). n.p. - not performed due to a language barrier. n.a. - not applicable

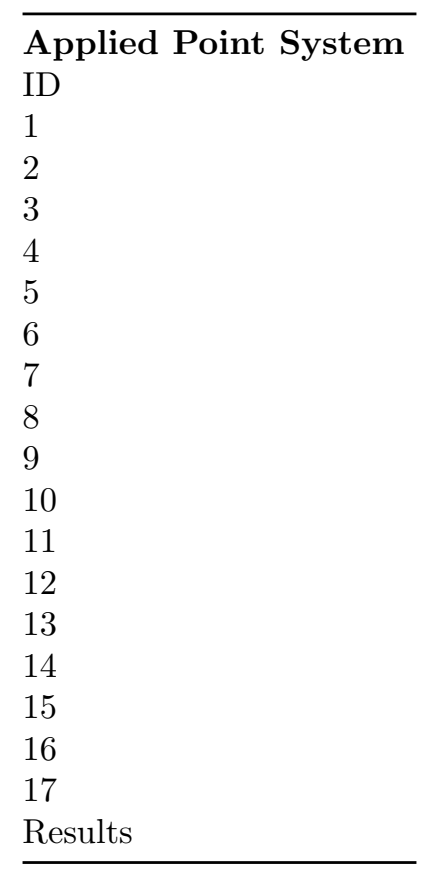

Table 4: Point system applied to the presented seventeen patients. Every column represents one of the categories and points given. Koos - Koos classification, Modiolus - extension (in connection to the Modiolus), Audio - audiometric results, summation of pure tone average and word recognition score) and PS eABR promontory stimulation electrically evoked auditory brainstem response. In total, there are four categories. Points reflects the sum of all points. Class is the resulting group each patient is categorized into, according to amount of points. Patient 1 did not undergo PS eABR, which does not allow for a complete classification. CI - cochlear implant: yes if they were provided with a CI, no if no CI was placed. N.p. - not performed

\section{Patient Demographics}




\begin{tabular}{|c|c|c|c|c|c|c|c|}
\hline ID & Age (y) & Sex & Side & 4-PTA & $\begin{array}{l}\text { WRS @ } \\
80 \mathrm{~dB}\end{array}$ & Contralateral & $4-\mathrm{PTA}_{\mathrm{C}}$ \\
\hline 1 & 47 & $\mathrm{f}$ & l & $77 \mathrm{~dB}$ & $20 \%$ & SSD & $6 \mathrm{~dB}$ \\
\hline 2 & 59 & $\mathrm{~m}$ & $\mathrm{r}$ & $68 \mathrm{~dB}$ & $40 \%$ & AHL & $54 \mathrm{~dB}$ \\
\hline 3 & 55 & $\mathrm{f}$ & l & $51 \mathrm{~dB}$ & $5 \%$ & SSD & $20 \mathrm{~dB}$ \\
\hline 4 & 74 & $\mathrm{~m}$ & $\mathrm{r}$ & $85 \mathrm{~dB}$ & $0 \%$ & AHL & $48 \mathrm{~dB}$ \\
\hline 5 & 42 & $\mathrm{f}$ & l & $68 \mathrm{~dB}$ & $0 \%$ & SSD & $8 \mathrm{~dB}$ \\
\hline 6 & 61 & $\mathrm{f}$ & 1 & $64 \mathrm{~dB}$ & $30 \%$ & SSD & $5 \mathrm{~dB}$ \\
\hline 7 & 69 & $\mathrm{f}$ & $\mathrm{r}$ & [?] $100 \mathrm{~dB}$ & $0 \%$ & [?] $100 \mathrm{~dB}$ & $65 \mathrm{~dB}$ \\
\hline 8 & 59 & $\mathrm{~m}$ & $\mathrm{r}$ & $60 \mathrm{~dB}$ & $0 \%$ & SSD & $21 \mathrm{~dB}$ \\
\hline 9 & 44 & $\mathrm{~m}$ & $\mathrm{r}$ & $74 \mathrm{~dB}$ & $0 \%$ & SSD & $6 \mathrm{~dB}$ \\
\hline 10 & 55 & $\mathrm{f}$ & $\mathrm{r}$ & $49 \mathrm{~dB}$ & $40 \%$ & SSD & $6 \mathrm{~dB}$ \\
\hline 11 & 56 & $\mathrm{f}$ & 1 & [?] $100 \mathrm{~dB}$ & $0 \%$ & SSD & $10 \mathrm{~dB}$ \\
\hline 12 & 60 & $\mathrm{f}$ & $\mathrm{r}$ & $87 \mathrm{~dB}$ & $0 \%$ & SSD & $16 \mathrm{~dB}$ \\
\hline 13 & 44 & $\mathrm{~m}$ & 1 & $75 \mathrm{~dB}$ & $0 \%$ & AHL & $55 \mathrm{~dB}$ \\
\hline 14 & 52 & $\mathrm{f}$ & 1 & [?] $100 \mathrm{~dB}$ & $0 \%$ & SSD & $25 \mathrm{~dB}$ \\
\hline 15 & 56 & $\mathrm{f}$ & l & $40 \mathrm{~dB}$ & $25 \%$ & SSD & $14 \mathrm{~dB}$ \\
\hline 16 & 62 & $\mathrm{f}$ & $\mathrm{r}$ & $64 \mathrm{~dB}$ & $0 \%$ & SSD & $20 \mathrm{~dB}$ \\
\hline 17 & 46 & $\mathrm{f}$ & 1 & [?] $100 \mathrm{~dB}$ & $0 \%$ & [?] $100 \mathrm{~dB}$ & $80 \mathrm{~dB}$ \\
\hline Total & $\begin{array}{l}55.4( \pm 8.9 \\
\mathrm{SD})\end{array}$ & $\begin{array}{l}\mathrm{m}=5 \mathrm{f}= \\
12\end{array}$ & $\mathrm{l}=9 \mathrm{r}=8$ & $\begin{array}{l}74 \mathrm{~dB}( \pm 19 \\
\mathrm{SD})\end{array}$ & $\begin{array}{l}9 \%( \pm 15 \\
\mathrm{SD})\end{array}$ & $\begin{array}{l}\mathrm{SSD}=12 \\
\mathrm{AHL}=3\end{array}$ & $\begin{array}{l}27 \mathrm{~dB} \\
( \pm 23 \mathrm{SD})\end{array}$ \\
\hline
\end{tabular}

Table 1: Age is given in years (y). Gender is described as $\mathrm{f}$ (female) or $\mathrm{m}$ (male). Puretone average (4-PTA) is calculated as average decibel (dB) hearing level (HL) at the frequencies $500 \mathrm{Hertz}(\mathrm{Hz}), 1000 \mathrm{~Hz}, 2000 \mathrm{~Hz}$ and $4000 \mathrm{~Hz}$. Word recognition scores (WRS) are calculated using Freiburg monosyllables at (@) 80 dB HL. Hearing of the contralateral side resulted in the diagnoses of single sided deaf (SSD), asymmetric hearing loss (AHL) or bilateral complete hearing loss larger than $100 \mathrm{~dB}$ HL ([?] $100 \mathrm{~dB})$. Contralateral 4-PTA = 4-PTAC

\section{Extension of Vestibular Schwannoma}

\begin{tabular}{ll}
\hline Points & Modiolus involvement \\
0 & No contact, no infiltration \\
1 & Contact with modiolus \\
2 & Infiltration of modiolus \\
3 & Transmodiolar extension \\
\hline
\end{tabular}

Table 2: Point system for extension of vestibular schwannoma towards the modiolus of the cochlear.

\section{Scoring System}

\begin{tabular}{lll}
\hline Category & Definition & Points \\
Koos Grading & Koos 4 & 0 \\
& Koos 3 & 1 \\
& Koos 2 & 2 \\
\multirow{4}{*}{ Extension } & Koos 1 & 3 \\
& Transmodiolar extension & 0 \\
& Infiltration of modiolus & 1
\end{tabular}




\begin{tabular}{lll} 
& Contact with modiolus & 2 \\
\multirow{5}{*}{ Hearing } & No contact, no infiltration of modiolus & 3 \\
& {$[?]$ 100 dB 4-PTA } & 0 \\
& $80<100 \mathrm{~dB}$ 4-PTA & 1 \\
& $<79 \mathrm{~dB}$ 4-PTA & 2 \\
PS EABR & $>0 \%$ monosyllables, any PTA & 3 \\
& No response & 0 \\
& Unclear wave V & 1 \\
& Stable wave V & 2 \\
& Class IV & $\mathbf{0}-\mathbf{3}$ \\
& Class III & $\mathbf{4}-\mathbf{5}$ \\
& Class II & $\mathbf{6}-\mathbf{8}$ \\
& Class I & $\mathbf{9 - 1 1}$ \\
\hline
\end{tabular}

Table 3: Scoring system to identify patients with higher chances of nerve integrity in case of VS resection. A certain amount (0-3) of points are given in four categories. Points are added up and patients are categorized to a certain class which reflects the probability of cochlear implantation after translabyrinthine vestibular schwannoma excision. Promontory stimulation eABR (PS EABR)

\section{Patient Outcomes}

\begin{tabular}{|c|c|c|c|c|c|c|c|}
\hline ID & Koos & CI & 6-month Follow-up & 6-month Follow-up & 6-month Follow-up & VII & $\begin{array}{l}\text { VII } \\
H B\end{array}$ \\
\hline & $1 / 2 / 3 / 4$ & yes/no & 4-PTA & WRS @ 65 dB & WRS @ 80 dB & HB - POD1 & $\mathrm{HB}-6 \mathrm{M}$ \\
\hline 1 & 2 & yes & $35 \mathrm{~dB}$ & $40 \%$ & $65 \%$ & 1 & 1 \\
\hline 2 & 1 & yes & $30 \mathrm{~dB}$ & $65 \%$ & $65 \%$ & 1 & 1 \\
\hline 3 & 3 & no & n.a. & n.a. & n.a. & 5 & 3 \\
\hline 4 & 3 & yes & $36 \mathrm{~dB}$ & $0 \%$ & $30 \%$ & 1 & 1 \\
\hline 5 & 2 & no & n.a. & n.a. & n.a. & 1 & 1 \\
\hline 6 & 1 & no & n.a. & n.a. & n.a. & 1 & 1 \\
\hline 7 & 2 & no & n.a. & n.a. & n.a. & 1 & 1 \\
\hline 8 & 4 & no & n.a. & n.a. & n.a. & 5 & 2 \\
\hline 9 & 2 & yes & $35 \mathrm{~dB}$ & $0 \%$ & $20 \%$ & 1 & 1 \\
\hline 10 & 2 & yes & $34 \mathrm{~dB}$ & $45 \%$ & $85 \%$ & 1 & 1 \\
\hline 11 & 2 & no & n.a. & n.a. & n.a. & 1 & 1 \\
\hline 12 & 2 & yes & $35 \mathrm{~dB}$ & $10 \%$ & $45 \%$ & 2 & 1 \\
\hline 13 & 2 & yes & $51 \mathrm{~dB}$ & n.p. & n.p. & 1 & 1 \\
\hline 14 & 1 & no & n.a. & n.a. & n.a. & 3 & 1 \\
\hline 15 & 2 & yes & $33 \mathrm{~dB}$ & $40 \%$ & $60 \%$ & 1 & 1 \\
\hline 16 & 1 & yes & $43 \mathrm{~dB}$ & $20 \%$ & $40 \%$ & 1 & 1 \\
\hline 17 & 2 & yes & $35 \mathrm{~dB}$ & $35 \%$ & $55 \%$ & 1 & 1 \\
\hline
\end{tabular}

Table 4: Outcomes of seventeen included patients. The second column shows the size and extension of the vestibular schwannoma according to Koos grading one to four. The third column shows which patients were provided with a cochlear implant (CI) (yes) and which not (no). Column four - Puretone average (4-PTA) in CI aided condition calculated as average decibel (dB) hearing level (HL) at the frequencies 500 Hertz (Hz), $1000 \mathrm{~Hz}, 2000 \mathrm{~Hz}$ and $4000 \mathrm{~Hz}$. Column five and six - word recognition scores (WRS) in CI aided condition are calculated using Freiburg monosyllables at (@) 65 and $80 \mathrm{~dB}$ HL. The last two columns show facial nerve function according to House Brackmann (HB) scale 1 to 6 on postoperative day one (POD 1) and at the 6 months follow-up appointment (6 Mo FU). n.p. - not performed due to a language barrier. n.a. - not applicable 


\section{Applied Point System}

\begin{tabular}{|c|c|c|c|c|c|c|c|}
\hline ID & Koos Points & Modiolus & Audio & PS eABR & Points & CIass & CI \\
\hline 1 & 2 & 2 & 3 & n.p. & [?] 7 & [?] II & yes \\
\hline 2 & 3 & 3 & 3 & 2 & 11 & I & yes \\
\hline 3 & 1 & 2 & 3 & 2 & 8 & II & no \\
\hline 4 & 1 & 3 & 1 & 2 & 7 & II & yes \\
\hline 5 & 2 & 2 & 2 & 2 & 8 & II & no \\
\hline 6 & 3 & 1 & 3 & 2 & 9 & I & no \\
\hline 7 & 2 & 0 & 0 & 1 & 3 & IV & no \\
\hline 8 & 0 & 1 & 2 & 2 & 5 & III & no \\
\hline 9 & 2 & 3 & 2 & 2 & 9 & I & yes \\
\hline 10 & 2 & 3 & 3 & 2 & 10 & I & yes \\
\hline 11 & 2 & 1 & 0 & 0 & 3 & IV & no \\
\hline 12 & 2 & 3 & 1 & 1 & 7 & II & yes \\
\hline 13 & 2 & 3 & 2 & 2 & 9 & I & yes \\
\hline 14 & 3 & 1 & 0 & 2 & 6 & II & no \\
\hline 15 & 2 & 3 & 3 & 2 & 10 & I & yes \\
\hline 16 & 3 & 2 & 2 & 2 & 9 & I & yes \\
\hline 17 & 2 & 2 & 0 & 2 & 6 & II & yes \\
\hline Results & $0-3$ & $0-3$ & $0-3$ & $0-2$ & $0-11$ & I - IV & yes/no \\
\hline
\end{tabular}

Table 5: Point system applied to the presented seventeen patients. Every column represents one of the categories and points given. Koos - Koos classification, Modiolus - extension (in connection to the Modiolus), Audio - audiometric results, summation of pure tone average and word recognition score) and PS eABR promontory stimulation electrically evoked auditory brainstem response. In total, there are four categories. Points reflects the sum of all points. Class is the resulting group each patient is categorized into, according to amount of points. Patient 1 did not undergo PS eABR, which does not allow for a complete classification. CI - cochlear implant: yes if they were provided with a CI, no if no CI was placed. 